\title{
A Novel Evolutionary Algorithm Inspired by Beans Dispersal
}

\author{
Xiaoming Zhang \\ Institute of Intelligent Machines, Chinese Academy of Sciences, Hefei, Anhui Province, 230031, China \\ University of Science and Technology of China, Hefei, Anhui Province, 230031, China \\ Bingyu Sun, Tao Mei, Rujing Wang \\ Institute of Intelligent Machines, Chinese Academy of Sciences, Hefei, Anhui Province, 230031, China
}

Received 6 October 2010

Accepted 4 November 2011

\begin{abstract}
Inspired by the transmission of beans in nature, a novel evolutionary algorithm-Bean Optimization Algorithm (BOA) is proposed in this paper. BOA is mainly based on the normal distribution which is an important continuous probability distribution of quantitative phenomena. Through simulating the self-adaptive phenomena of plant, BOA is designed for solving continuous optimization problems. We also analyze the global convergence of BOA by using the Solis and Wets' research results. The conclusion is that BOA can converge to the global optimization solution with probability one. In order to validate its effectiveness, BOA is tested against benchmark functions. And its performance is also compared with that of particle swarm optimization (PSO) algorithm. The experimental results show that BOA has competitive performance to PSO in terms of accuracy and convergence speed on the explored tests and stands out as a promising alternative to existing optimization methods for engineering designs or applications.
\end{abstract}

Keywords: evolutionary algorithm, swarm intelligence, particle swarm optimization, bean optimization algorithm.

\section{Introduction}

From the middle of the last century, biology simulation has been an important component in computer science and artificial intelligence. Many intelligent algorithms are developed by imitating natural selection and evolution directly, such as Genetic Algorithm (GA) [1], Particle Swarm Optimization Algorithm (PSO) [5], Ant Colony Algorithm [2], Immune Algorithm [7], Free Search [8], and Human Evolutionary Model [9]. Because the structure of nature biology is complex and sophisticated, the nature-inspired algorithms always have a high degree of adaptive capacity and strong collaborative capability both in the evolutions and behaviors. Through collaboration, they can get the best environment for survival. Therefore, most of the nature- inspired algorithms have the character of self-organizing, self-adaptive and self-learning. When solving some complex problems which the traditional optimization algorithm can not solve easily, the nature-inspired optimization algorithms have its own unique advantages. At present, nature-inspired optimization algorithms have been used to solve complex optimization problems in many fields successfully, such as task assignment [3], classification [4], and gene selection [6].

Inspired by the transmission mode of beans, we propose a novel evolutionary algorithm namely Bean Optimization Algorithm (BOA) which can be used to solve continuous optimization problems by simulating the adaptive phenomenon of plants in nature.

In the rest of this paper, we will first introduce the basic idea of BOA and the details of its implementation in 
Section 2. Next, we will carry out the convergence analysis of BOA in Section 3. Then we will present the experiments and result analysis of the proposed BOA in Section 4. The paper is concluded in Section 5.

\section{Bean Optimization Algorithm}

\subsection{Basic Idea of $\mathrm{BOA}$}

As we know, there are many different seed transmission modes in nature. For example, most of the legume explore scattering mode. The scattering mode can be described by the following scenes. When the beans are ripe, their skin becomes dry and hard in the sun's radiation. Then their skin bursts and most of the beans are ejected to the region around the plant. Also, some beans may move or fly far away from the plant for some reasons, for example, a bean can be carried by some animals or wind. Assume that all beans will then grow and develop in the region which they land on. There is no doubt that some beans will grow to be very strong plants and produce lots of seeds where the region they land on is very fertile. On the contrary, some may soon be phased out because they are not suitable for the region which they grow in. After a long time, a large number of beans will be gathered in the fertile region, and most of those beans in the infertile region will be disappeared. Inspired by this phenomenon, in this paper, the domain of optimization problem will be treated as a land and the position of the target point is set in the most fertile position. The degree of the region's fertility is determined in accordance with the value of the objective function. It can be given a concrete example: when a packet of beans are randomly spread to the land, the probability of their growing vigorously and producing more offspring will be great if the beans fall to the fertile region. If the beans fall to the barren region, they will likely be eliminated. After the beans have evolved for lots of generations, there might be one or several plants growing in the most fertile region. In $\mathrm{BOA}$, the evolution process is abstracted. It can be described as such phenomenon: in every new generation, most of the young plants emerge around several most powerful old plants (we call the powerful old plants father beans).

\subsection{Algorithm Design}

In BOA, the position of individual beans are expressed with real number vector like

$$
X=\left(x_{1}, x_{2}, x_{3}, \ldots, x_{n}\right),
$$

where $n$ is the dimension which is determined by the scale of problem to be resolved. The bean group is composed of a large number of beans. And the size of the bean group can be adjusted according to the practical situations. In addition, beans are sown to the region which is defined by the problem. Father beans are those beans whose fitness values are larger than that of most of the others. In BOA, the parameters of distribution and the number of descendant beans will be set according to their father bean's fitness value. That is to say, the larger is the value of father bean's fitness, the larger is the number of its descendants and the closer is the distance between them. Otherwise, the number of its descendants would be fewer and its descendants would have a more random distribution. The basic equation of beans is shown as followings:

$X[i]=\left\{\begin{array}{l}X[i], \text { if } X[i] \text { is a father bean } \\ \operatorname{Distribution}\left(X_{m b}\right) \times A, \text { if } X[i] \text { is not a father bean }\end{array}\right.$

In the above equation (1), $X[i]$ is the position of bean $i$ and $X_{m b}$ is the position of father bean of bean $i$. Distribution $\left(X_{m b}\right)$ is the distribution function of father bean in order to get the positions of its descendants. Parameter $A$ can be set according to the range of the problem to be resolved.

Normal distribution is an important family of continuous probability distributions, applicable in many fields. The importance of the normal distribution as a model of quantitative phenomena in the natural is due in part to the central limit theorem. It is far more common to describe a normal distribution by its mean $\mu$ and variance $\sigma^{2}$. The probability density function in a convenient standard form is shown as follows:

$$
f(X)=\frac{1}{\sigma \sqrt{2 \pi}} e^{-(X-\mu)^{2} /\left(2 \sigma^{2}\right)},-\infty<X<+\infty
$$

Many measurements, ranging from psychological to physical phenomena can be approximated, to varying degrees, by the normal distribution. While the mechanisms underlying these phenomena are often unknown, the use of the normal model can be theoretically justified by assuming that many small, independent effects are additively contributing to each observation. In this paper, we adopt normal distributions 
as the distribution functions of beans. And the mean and variance of every distribution functions are set according to the fitness of the father beans.

The distribution of descendants around their father beans is shown in Fig. 1.

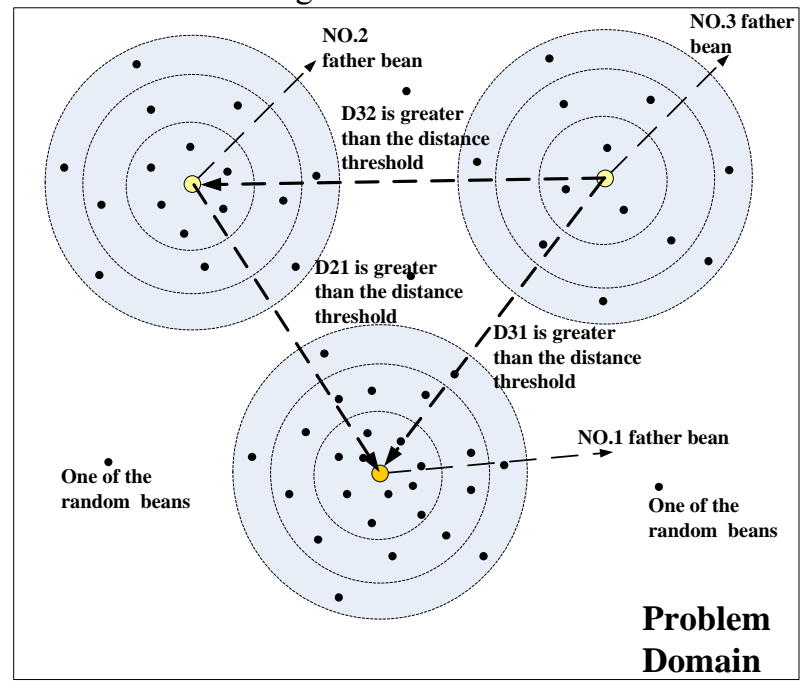

Fig.1. Sketch Map of Beans Distribution

We also define the threshold of population variation. If the population variation happens, the distribution of some beans will not follow the equation discussed above. They will choose their positions randomly and that will reinforce the global optimization performance. When the descendant beans finished locating, their fitness values will be evaluated. The beans with the most optimal fitness value will be selected as the candidates of next generation father beans. The father beans candidates should also satisfy the condition that the distance between every two father beans should be larger than the distance threshold. This condition assures that the father beans can have a fine distributing to avoid premature convergence and to enhance the performance for global optimization. If all the conditions can be satisfied, the candidate bean can be set as a father bean of the next generation. The flow chart of father beans selection is shown in Fig. 2 .

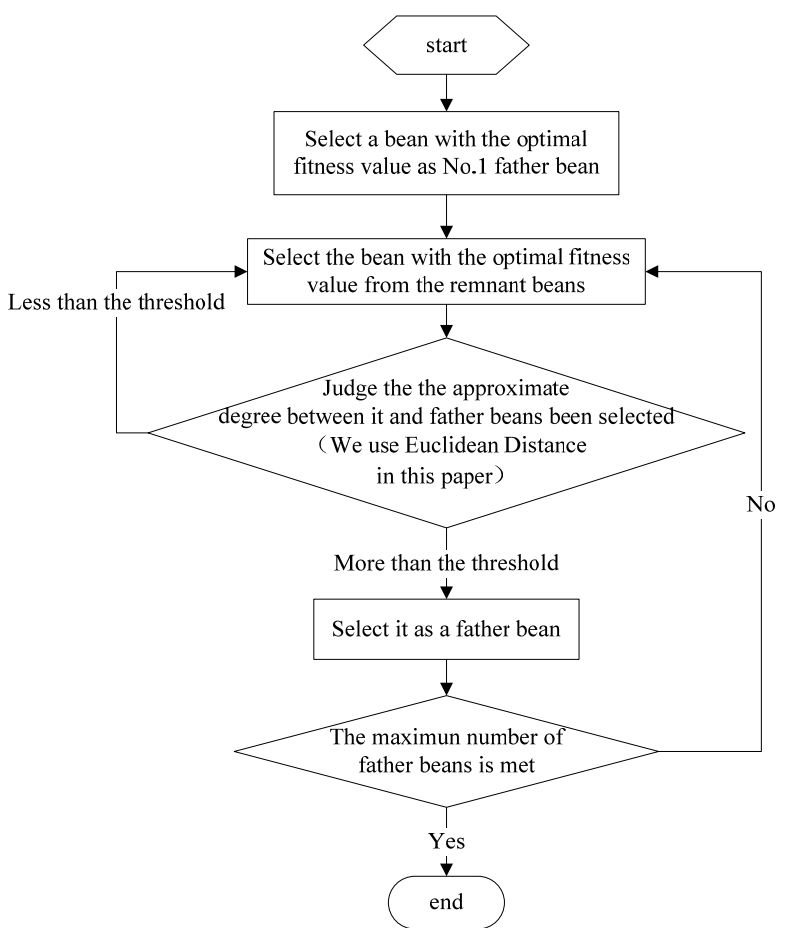

Fig.2. The Selection of Father Beans

According to the evolution equations of BOA, beans are sown in the radiate area of their father beans in every generation. Evolution will go on until the desired optimization result is got or the maximum generation is met. The process of the BOA based on normal distribution is listed in table 1 .

Table1. The Basic Process of BOA

1. Initialization parameters (problem domain, the threshold of
the approximate degree between father beans, etc.)
2.Population of beans initialization(Randomly initialize
positions)
3.Calculate the fitness value
4.Select the No.1 father bean
5. While(not meet the termination condition of the Algorithm)
6. Select the father beans(except No.1 father bean)
7. For(each individual of the population)
8. If (the NumPop is not the index of father beans)
9. $\quad$ pop(NumPop, :) = normrnd(FthPop,Variance)
10. Else If (random number is bigger than the threshold
11. population variation)
12. $\quad$ End If
13. End For
14. Population update
15. No.1 father bean update
16.End While
17. Output the No.1 father bean and its fitness




\section{Convergence Analysis}

BOA is a kind of stochastic optimization algorithm. Thus, we can use the theory about how a kind of stochastic algorithm can converge to the global optimization solution with probability one [11]. We use the theory which Solis and Wets have proved to be right [10]. Here, we list their main conclusions:

(H1) D s.t. $\left\{f\left(x_{k}\right)\right\}_{k=0}^{\infty}$ nonincreasing,

$$
\begin{gathered}
f(D(x, \xi)) \leq f(x) \\
\xi \in A \Rightarrow f(D(x, \xi)) \leq \min \{f(x), f(\xi)\}
\end{gathered}
$$

$\mathrm{D}$ is a function that constructs a solution to the problem. $\xi$ is a random vector based on probability space $\left(R^{n}, B\right.$, $\left.\mu_{k}\right) . f$ is the objective function. $A$ is the search space.

(H2) Zero probability of repeatedly missing any positive-volume subset of $S$.

$$
\forall B \subseteq A, \quad \text { s.t. } v(B)>0, \sum_{k=0}^{\infty}\left(1-u_{k}(B)\right)=0
$$

$B$ is the $\sigma$-algebra of subset of $R^{n} . u_{k}(B)$ is a probability measure on $B$.

Theorem 1 (Global Search Convergence Theorem). Suppose $f$ measurable, $S \subseteq R^{n}$ measurable, (H1), (H2), and $\left\{x^{k}\right\}_{k=0}^{\infty}$ generated by the algorithm. Then

$$
\lim _{k \rightarrow \infty} P\left(x^{k} \in R_{\varepsilon, M}\right)=1
$$

where $P\left(x^{k} \in R_{\varepsilon, M}\right)$ is the probability that at step $k$, the point $x^{k}$ generated by the algorithm is in $R_{\varepsilon, M}$ (the set of global optimal points).

Then we just need to analyze whether the BOA accords with Theorem 1 as stated above. In order to do this, we should analyze the two hypothesizes $(\mathrm{H} 1)$ and ( $\mathrm{H} 2$ ) first. BOA returns the position of No.1 father bean. Let $t$ be the number of the generation of BOA. What BOA returns is the position of No.1 father bean in generation $t\left(X_{g}\right)$. Let $X(t)$ be the position of one bean in generation $t$. $f(x)$ is the fitness evaluation function. So we can define the function $D$ in (H1) as follows:

$$
D\left(X_{g}, X(t)\right)=\left\{\begin{array}{l}
X_{g}, f\left(X_{g}\right) \leq f(X(t)), \\
X(t), f\left(X_{g}\right)>f(X(t)),
\end{array}\right.
$$

It is easy to see that BOA can satisfy the hypothesis (H1).

In order to prove that BOA also satisfy the hypothesis (H2), we need to prove that the union of the sample space of beans group contains $S$.

So we make the analysis in connection with this. Let the number of father beans be $N . S_{i}$ is the radiate range of father bean $i$. Then the union of the beans' supporting set is $\bigcup_{i=1}^{n} S_{i}$.

If $S_{i}$ is set to be large enough to cover the problem region, we can get $S=\bigcup_{i=1}^{n} S_{i}$. In fact it is easy to realize by just set $S_{i}$ extend to the region border. So we can see that BOA can satisfy the hypothesis (H2). That is to say that BOA accords with the Theorem 1.

Sum up all the analysis, BOA can converge to the global optimization solution with probability one.

\section{Experiment and analysis}

The experiments were carried out on a PC with a 2.10$\mathrm{GHz}$ Intel Processor and 2.0-GB RAM. All the programs were written and executed in MATLAB 7.0. The operating system was Microsoft Windows XP.

\subsection{Benchmark Functions}

Three well-known benchmark functions are employed to evaluate the performance of the BOA algorithm. They are given in Table 2. As PSO algorithm is generally found to perform better than other algorithms (GA, MA, PSO, ACO, and SFL) in terms of success rate and solution quality [12], we will compare the performance of BOA with PSO.

Table 2 Benchmark Functions

\begin{tabular}{|c|c|c|c|}
\hline Function Name & Rosenbrock & Schaffer's F6 & Rastrigin \\
\hline Function No. & F5 & F6 & F9 \\
\hline $\begin{array}{c}\text { Mathematical } \\
\text { Representation } \\
(f(\mathrm{X})=)\end{array}$ & $\sum_{i=1}^{n-1}\left[100\left(x_{i+1}-x_{i}^{2}\right)^{2}+\left(1-x_{i}\right)^{2}\right]$ & $0.5+\frac{\left(\sin \sqrt{x^{2}+y^{2}}\right)-0.5}{\left(1.0+0.001\left(x^{2}+y^{2}\right)\right)^{2}}$ & $\sum_{i=1}^{n}\left[x_{i}^{2}-10 * \cos \left(2 \pi * x_{i}\right)+10\right]$ \\
\hline Initial Range & {$[-30,30]$} & 0 & {$[-5.12,5.12]$} \\
\hline $\begin{array}{c}\text { Global Optimization } \\
\text { Solution }\end{array}$ & 0 & $0,100]$ & 0 \\
\hline
\end{tabular}




\subsection{Experimental Settings}

Table 3 Experimental Settings of BOA

\begin{tabular}{|c|c|c|c|}
\hline $\begin{array}{l}\text { Function } \\
\text { Name }\end{array}$ & $\begin{array}{l}\text { Distance } \\
\text { Threshold }\end{array}$ & $\begin{array}{l}\text { The Distribution of Future } \\
\text { Generations }\end{array}$ & Commentary \\
\hline Rosenbrock & $\begin{array}{l}\text { D21 }=1.2 \\
\text { D31 }=1.2 \\
\text { D32 }=1.2\end{array}$ & \multirow{3}{*}{$\begin{array}{l}70 \% \text { of beans are in } \\
\text { accordance with distribution } \\
\text { normrnd(FthPop }(1,:), 1) \text {. } \\
20 \% \text { of beans are in } \\
\text { accordance with distribution } \\
\text { normrnd(FthPop }(2,:), 1.2) \text {. } \\
10 \% \text { of beans are in } \\
\text { accordance with distribution } \\
\text { normrnd(FthPop }(3,:), 1.5) \text {. }\end{array}$} & $\begin{array}{l}\text { 1. The scale of the } \\
\text { population of beans is } 50 . \\
\text { 2. The number of father } \\
\text { beans is } 3 \text {. }\end{array}$ \\
\hline $\begin{array}{l}\text { Schaffer's } \\
\text { F6 }\end{array}$ & $\begin{array}{l}\text { D21 }=6.0 \\
\text { D31 }=9.0 \\
\text { D32 }=9.0\end{array}$ & & $\begin{array}{l}\text { 3. FthPop(i, :) is the } \\
\text { position of father bean i. } \\
i=1,2,3 \text {. }\end{array}$ \\
\hline Rastrigin & $\begin{array}{l}\text { D21 }=3.0 \\
\text { D31 }=5.0 \\
\text { D32 }=5.0\end{array}$ & & $\begin{array}{l}\text { 4. } \mathrm{D}_{\mathrm{ij}} \text { is the distance } \\
\text { threshold between father } \\
\text { bean } \mathrm{i} \text { and father bean } \mathrm{j} \text {. }\end{array}$ \\
\hline
\end{tabular}

Table 4 Experimental Settings of PSO

\begin{tabular}{|c|c|c|}
\hline $\begin{array}{c}\text { Function } \\
\text { Name }\end{array}$ & {$[$ Vmax,Vmin $]$} & Commentary \\
\hline Rosenbrock & {$[-0.5,0.5]$} & $\begin{array}{c}\text { 1. The scale of the population } \\
\text { of particles is } 50 .\end{array}$ \\
\cline { 1 - 1 } $\begin{array}{c}\text { Schaffer's } \\
\text { F6 }\end{array}$ & {$[-1,1]$} & $\begin{array}{c}\text { 2. The acceleration factors of } \\
\text { PSO is: } \\
\mathrm{c} 1=\mathrm{c} 2=1.49445\end{array}$ \\
\hline Rastrigin & {$[-1,1]$} & \\
\hline
\end{tabular}

\subsection{Experimental Results}

Table 5 Experimental Results

\begin{tabular}{|c|c|c|c|}
\hline $\begin{array}{c}\text { Function } \\
\text { Name }\end{array}$ & Algorithm & Mean Result & STD \\
\hline \multirow{2}{*}{ Rosenbrock } & BOA & $\mathbf{4 5 . 9 5 2 8}$ & $\mathbf{1 4 . 9 2 4 8}$ \\
\cline { 2 - 4 } & PSO & 90.8311 & 64.36 \\
\hline \multirow{2}{*}{ Schaffer's F6 } & BOA & $\mathbf{5 . 4 8 4 7 e - 0 0 4}$ & $\mathbf{0 . 0 0 1 9}$ \\
\cline { 2 - 4 } & PSO & 0.0025 & 0.0043 \\
\hline \multirow{2}{*}{ Rastrigin } & BOA & $\mathbf{1 5 . 0 1 2 0}$ & 14.0819 \\
\cline { 2 - 4 } & PSO & 37.5870 & $\mathbf{8 . 7 2 2 9}$ \\
\hline
\end{tabular}


The maximum number of BOA iterations for the benchmark functions are set to be 500 for both PSO and BOA. Table 5 lists the mean results and standard deviations of the best results found in 50 runs of BOA on test functions. The results generated from PSO are also tabulated in Table 5 in comparison with the results generated by BOA.

The convergence figures and stem charts based on the results of the experiments are shown in Fig.3-Fig.11. In the following convergence figures, the blue curves are the convergence curves of BOA in every run time. The black one is the mean convergence curve of BOA. The red curves are the convergence curves of PSO in every run time. The green one is the mean convergence curve of PSO. In order to clarify the convergence figures, the scale of horizontal axis is set to be "Linear" and the scale of vertical axis is set to be "Log".

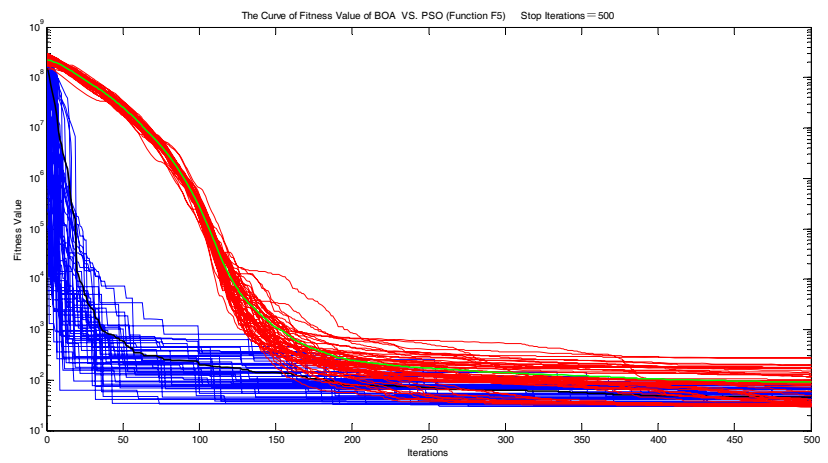

Fig.3. This is the convergence figure of BOA compared with PSO when solving Rosenbrock (F5) in 30 dimensions.

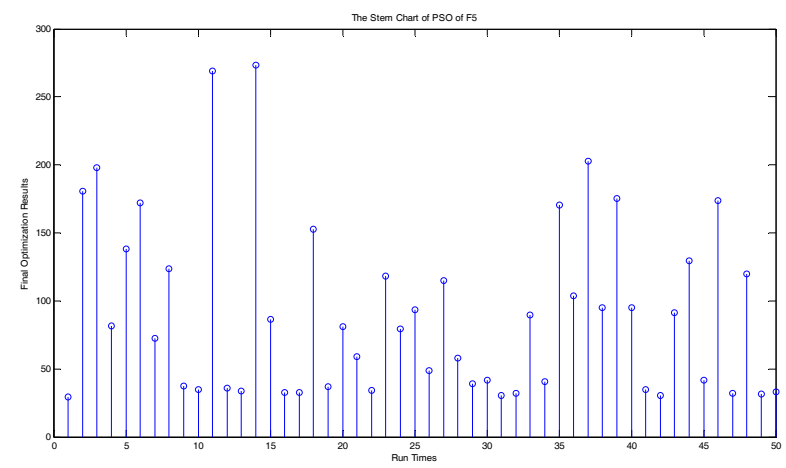

Fig.4. The figure is the stem chart of 50 final optimization results of PSO when solving Rosenbrock (F5) in 30 dimensions.

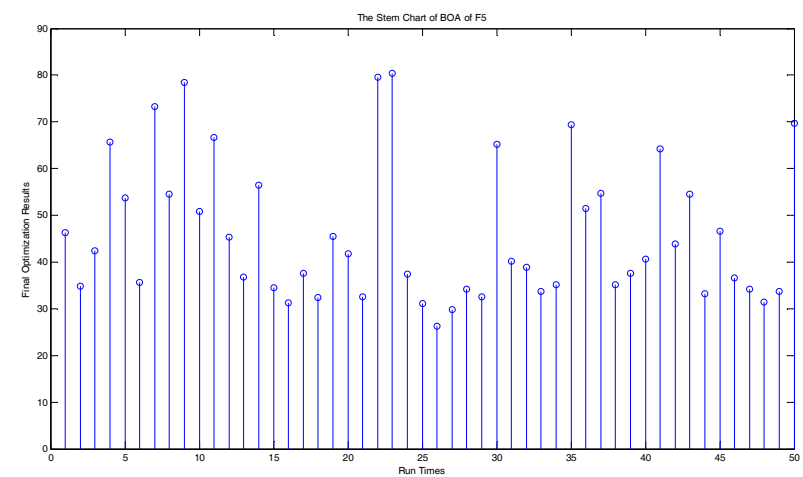

Fig.5. The figure is the stem chart of 50 final optimization results of BOA when solving Rosenbrock (F5) in 30 dimensions.

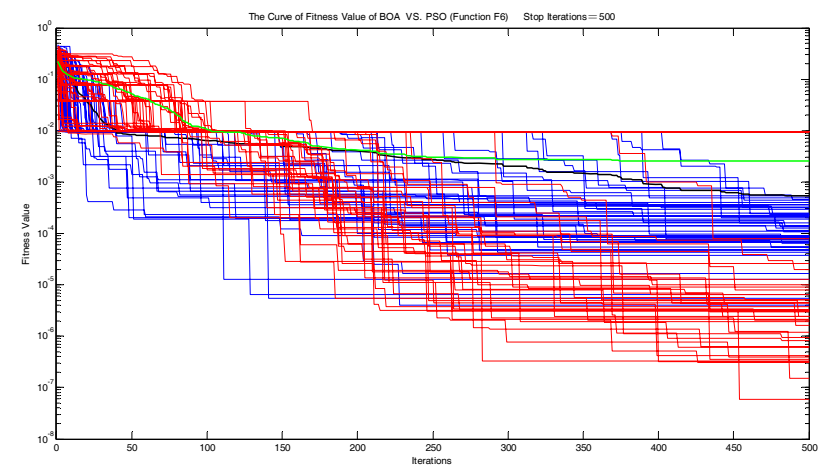

Fig.6. This is the convergence figure of BOA compared with PSO when solving Schaffer's F6 (F6) in 2 dimensions.

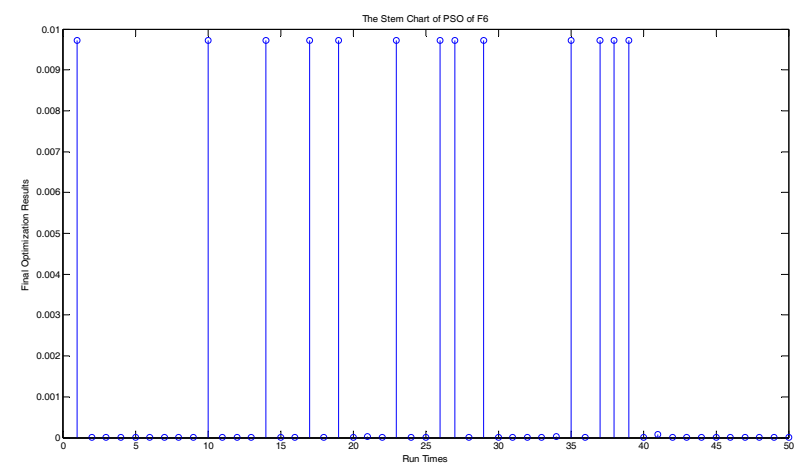

Fig.7. The figure is the stem chart of 50 final optimization results of PSO when solving Schaffer's F6 (F6) in 2 dimensions. 


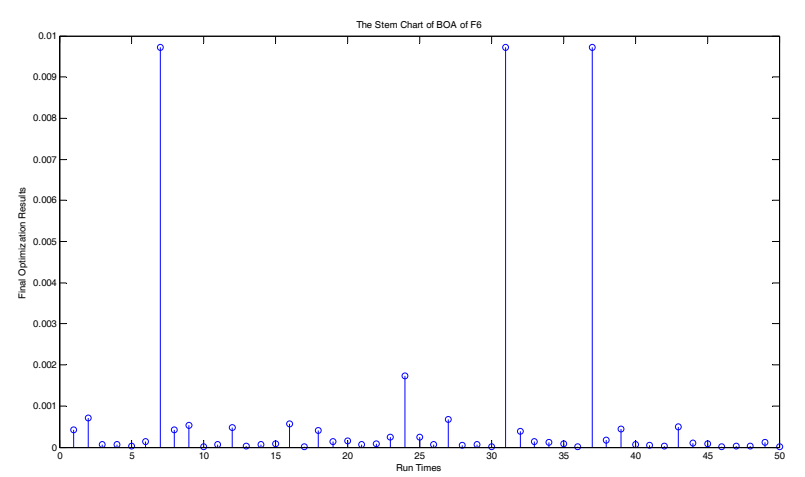

Fig.8. The figure is the stem chart of 50 final optimization results of BOA when solving Schaffer's F6 (F6) in 2 dimensions.

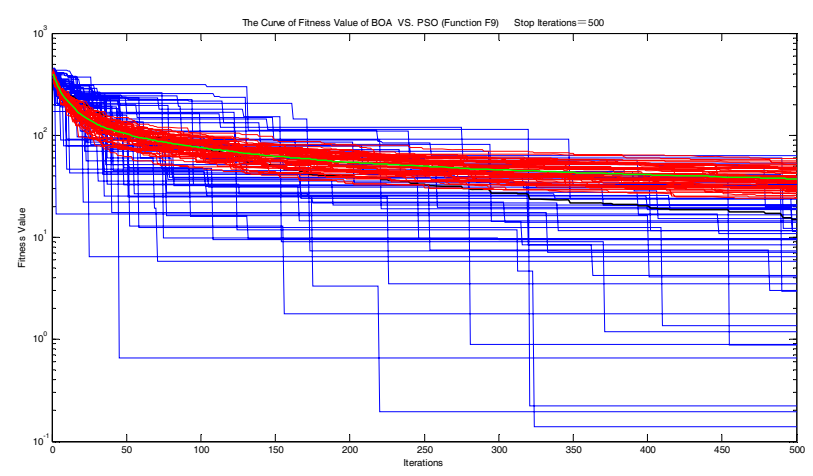

Fig.9. This is the convergence figure of BOA compared with PSO when solving Rastrigin (F9) in 30 dimensions.

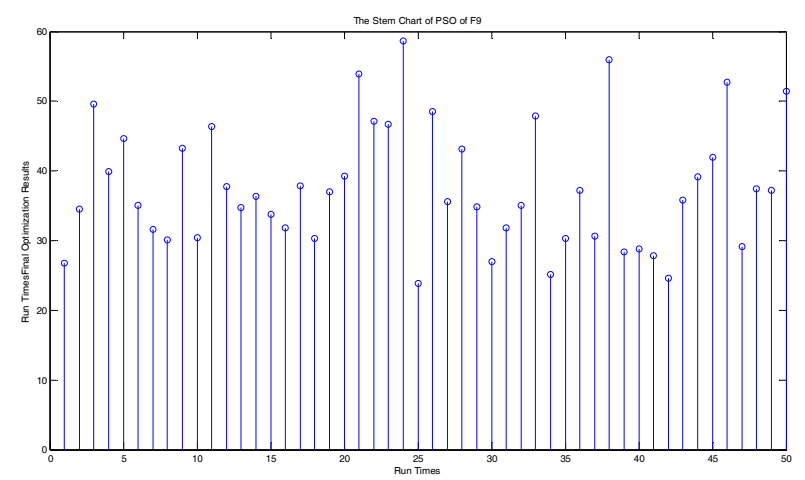

Fig.10. The figure is the stem chart of final optimization results of PSO of 50 run times when solving Rastrigin (F9) in 30 dimensions.

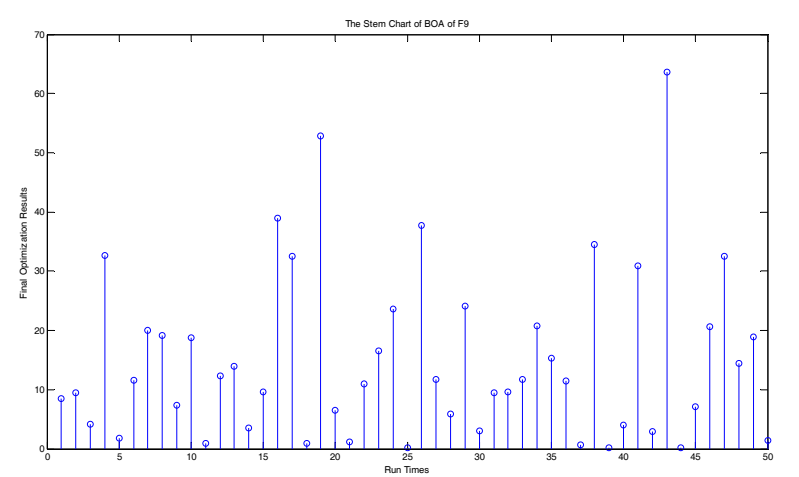

Fig.11. The figure is the stem chart of 50 final optimization results of BOA when solving Rastrigin (F9) in 30 dimensions..

\subsection{Results Analysis}

From the results of the above experiments, we can see that all of the best mean results are got by BOA. That is to say, BOA has better performance than PSO in the aspect of solution quality. Except in function Rastrigin, the STD values of BOA is less than that of PSO. That means BOA is more stable than PSO in the above experiments. We also can see that the convergence speed of BOA is better than that of PSO from the convergence curves.

However, the ease-of-use of BOA is not better than PSO algorithm. For different problem, BOA needs to be set different parameters or different distribution functions, such as, the number of father beans and Distribution $\left(X_{m b}\right)$. Inappropriate parameter settings of BOA will impact on the performance of the algorithm.

\section{Conclusions and Outlook}

In this paper, a novel evolutionary algorithm - Bean Optimization Algorithm is presented and its global optimization performance is analyzed. BOA is designed for solving continuous optimization problems and it can converge to the global optimization solution with probability one. In the experiments of function optimization, results show that BOA has competitive performance to PSO in terms of accuracy and convergence speed on the explored tests and stands out as a promising alternative to existing optimization methods for engineering designs or applications. Because the research on BOA is just at a preliminary stage, some related methods of parameter setting need to be studied in-depth. In the future, the research on the algorithm will focus on the dissemination of father beans and the distributions of descendant beans in order to improve the performance of BOA. 


\section{Acknowledgements}

This work was supported by the National Science Foundation of China under Grant 61203373, 91024008, and the Knowledge Innovation Program of the Chinese Academy of Sciences.

\section{References}

1. J. H. Holland. Adaptation in Natural and Artificial Systems. University of Michigan Press. Ann Arbor, (1975).

2. F. Moyson, B. Manderick. The Collective Behaviour of Ants: an Example of Self-Organization in Massive Parallelism. Proceedings of the AAAI Spring Symposium on Parallel Models of Intelligence, Stanford, California, (1988).

3. Shinn-Ying Ho, Hung-Sui Lin, Weei-Hurng Liauh, Shinn-Jang Ho. OPSO: Orthogonal Particle Swarm Optimization and Its Application to Task Assignment Problems. IEEE Transactions on Systems, Man and Cybernetics, Part A, 38(2), pp.288-298, (2008).

4. Souda T., Silva A., Neves A. Particle Swarm based Data Mining Algorithms for classification task. Parallel Computing, 30(5), pp.767-783, (2004).

5. Kennedy J, Eberhart R C. Particle swarm optimization. In Proceedings of the IEEE International Conference on Neural Networks, pp.1942-1948, (1995).

6. Shutao Li, Xixian Wu, Mingkui Tan Gene selection using hybrid particle swarm optimization and genetic algorithm. Soft Computing, Vol.12(11), pp.1039-1048, (2008).

7. Wang Lei, Pan Jin, Jiao Li-cheng. The Immune Algorithm. Acta Electronica Sinica, Vol.28(7), pp.74-78, (2000).

8. Kalin Penev, Guy Littlefair. Free Search-a comparative analysis. Information Sciences, Vol.172(1-2), pp.173193, (2005).

9. Oscar Montiel, Oscar Castillo, Patricia Melin, Antonio Rodríguez Díaz, Roberto Sepúlved. Human evolutionary model: A new approach to optimization. Information Sciences, Vol.177(10), pp. 2075-2098, (2007).

10. Solis F., Wets R. Minimization by Random Search Techniques. Mathematics of Operations Research, Vol.6(1), pp.19-30, (1998).

11. Zeng J.C., Cui Z.H. A Guaranteed Global Convergence Particle Swarm Optimizer. Journal of Computer Research and Development, Vol.41(8), pp. 1333-1338, (2004).

12. Emad Elbeltagia, Tarek Hegazyb, Donald Grierson. Comparison among five evolutionary-based optimization algorithms. Advanced Engineering Informatics, Vol.19(1), pp.43-53, (2005). 